\title{
Nanoscale Surface and Sub-Surface Chemical Analysis of SiGe Nanowires
}

\author{
W. Hourani ${ }^{1}$, E. Martinez ${ }^{1}$, P. Periwal ${ }^{2}$, G. Patriarche ${ }^{3}$, F. Bassani ${ }^{2}$, J.M. Fabbri ${ }^{1}$, T. Baron ${ }^{2}$ \\ ${ }^{1}$ CEA, LETI, MINATEC Campus, 17 rue des Martyrs, 38054 Grenoble Cedex 9, France \\ ${ }^{2}$ CNRS, LTM, 17 rue des Martyrs, 38054 Grenoble, France \\ ${ }^{3}$ Laboratoire de Photonique et de Nanostructures-CNRS, Route de Nozay, 91460 Marcoussis, France
}

$\mathrm{Si} / \mathrm{Si}_{1-\mathrm{x}} \mathrm{Ge}_{\mathrm{x}}$ axial heterostructured nanowires (hNW) are under investigation for downscaling of metaloxide-semiconductor field-effect transistors (MOSFETs). New architectures based on vertically aligned nanowires tunnel-FETs are promising candidates for reduced power dissipation and low voltage operation [1]. The axial growth of lattice mismatched heterostructures would allow band-gap engineering along the charge transport direction. Adequate control of the chemical composition along axial and radial directions is of upmost importance for this band-gap engineering.

Scanning transmission electron microscopy (STEM) is combined with electron dispersive spectroscopy (EDS) to check the chemical composition of these nanostructures. In this work, a surface technique such as scanning Auger microscopy (SAM) is used to expand and complement STEM/EDS analysis. Radial composition heterogeneities are highlighted combining high spatial resolution $(\sim 10 \mathrm{~nm})$ and quantitative elemental characterization [2].

$\mathrm{Si} / \mathrm{Si}_{1-\mathrm{x}} \mathrm{Ge}_{\mathrm{x}}$ hNWs were grown via the vapor-liquid-solid (VLS) growth mechanism in a chemical vapor deposition (CVD) reactor at $450^{\circ} \mathrm{C}$ using $\mathrm{Au}$ as catalyst. Three different segments with increasing Ge/Si ratio, starting from the bottom up to the catalyst, were fabricated $\left(\mathrm{Si}_{0.7} \mathrm{Ge}_{0.3}, \mathrm{Si}_{0.4} \mathrm{Ge}_{0.6}\right.$ and $\left.\mathrm{Si}_{0.2} \mathrm{Ge}_{0.8}\right)$ by changing the fluxes of precursor gases $\left(\mathrm{GeH}_{4}\right.$ and $\left.\mathrm{SiH}_{4}\right)$. Their chemical compositions were measured by STEM/EDS, XRD and Raman spectroscopy on reference single composition nanowires (see figure $1)$.

The Auger experiments were carried out using a PHI 700Xi Auger nanoprobe equipped with a coaxial cylindrical mirror analyzer (CMA) with energy resolution of $0.5 \%$ and high sensitivity (ultimate detection limit $\sim 0.1 \%$ ). Measurements were performed with an incident electron beam at $20 \mathrm{kV}$ and $1 \mathrm{nA}$ (probe size $\sim 20 \mathrm{~nm}$ ). The NWs were dispersed on a $100 \mathrm{~nm}$-thick TiN layer to avoid backscattered electron beam induced Auger electrons.

Prior to the Auger analysis, an adequate surface preparation is needed. Chemical etching is used to remove native oxide by deposition of a $1 \% \mathrm{HF}$ aqueous droplet on the surface during $2 \mathrm{~min}$. The Auger survey spectra measured before and after this surface treatment are presented in figure 2 . The oxygen O KLL peak has almost disappeared after treatment. A subsequent low energy $(500 \mathrm{eV})$ argon ion sputtering is performed during $5 \mathrm{~min}$ with Zalar rotation to remove the hydrocarbon surface contamination (see figure 2).

Quantitative analysis of the chemical composition is obtained by using tabulated sensitivity factors (0.071 for Si KLL and 0.149 for Ge LMM). In a previous work [3], we have shown that these factors are valid up to $20 \mathrm{kV}$ for bulk SiGe multilayers. The Auger peak intensities are extracted from the maximum amplitude of Auger spectra after numerical derivation to remove the background signal.

Localized Auger electron spectroscopy (AES) measurements are performed at the interface between the $\mathrm{Si}_{0.7} \mathrm{Ge}_{0.3}$ and $\mathrm{Si}_{0.4} \mathrm{Ge}_{0.6}$ regions (not shown here). The surface chemical composition is measured to be $\mathrm{Si}_{0.2} \mathrm{Ge}_{0.8}$ and $\mathrm{Si}_{0.1} \mathrm{Ge}_{0.9}$ respectively. This is confirmed by an Auger analysis performed in line-scan mode along the axial direction between zones $\mathrm{B}$ and $\mathrm{C}$ (see figure 3 ). This confirms the phenomenon of 
surface/bulk difference which is attributed to Ge surface segregation and to additional Ge deposition at the surface induced by radial growth [4]. The difference is even more important for the first segment which undergoes long exposure to $\mathrm{GeH}_{4}$ during the growth of second and third sections.

This work was partially performed at the Nano-Characterization platform (PFNC) of MINATEC. Further measurements combining localized AES analysis with argon ion sputtering will be performed to obtain the elemental depth composition of such nanostructures ( $\mathrm{Si}$ and Ge profiles) and measure the variations along both axial and radial directions.

\section{References:}

[1] G. Rosaz et al., Appl. Phys. Lett. 99, 193107 (2011).

[2] E. Martinez et al., J. Electron Spectrosc. Relat. Phenom., in press (2014).

[3] P. K. Yadav et al., IOP Conf. Series: Mat. Sci. \& Eng., 41, 012020 (2012).

[4] D. E. Perea et al., Journal of Solid State Chemistry, 181 (2008) 1642- 1649.
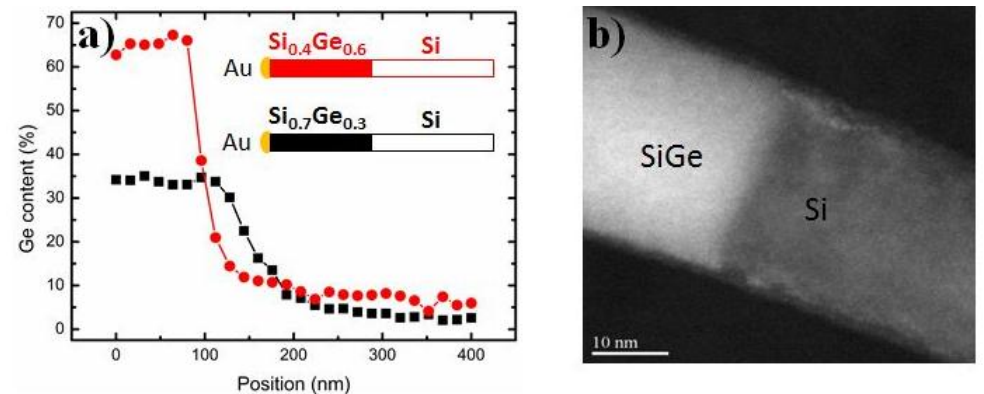

Figure 1. a) EDS showing the chemical composition of two SiGe nanowires with two different concentrations.

b) HAADF-STEM image of $\mathrm{Si} / \mathrm{SiGe}$ segment.

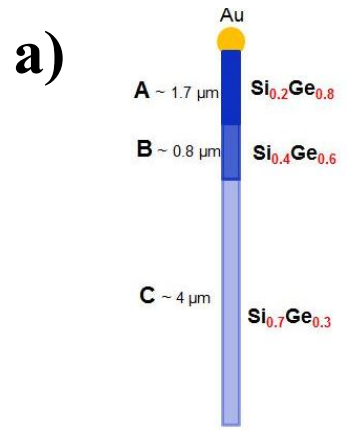

Figure 2. a) Schematic representation of the SiGe under study. b) Auger survey spectra measured on the SiGe nanowire before and after surface treatments.

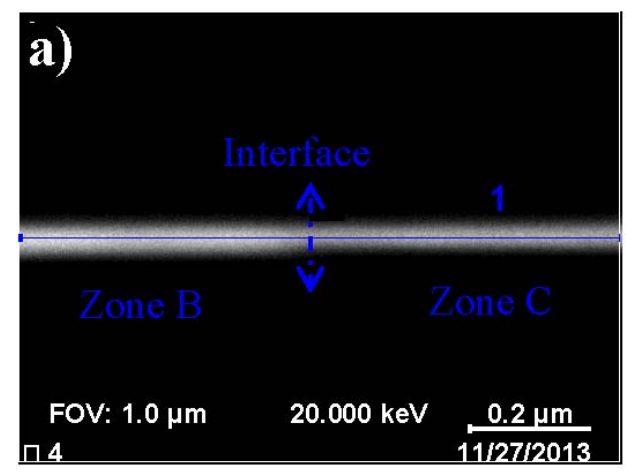

Figure 3. a) SEM image of the SiGe nanowire showing the interface between the two zones B and C. b) Atomic concentration profiles of $\mathrm{Si}$ and $\mathrm{Ge}$ along the $\mathrm{SiGe}$ nanowire between zones $\mathrm{B}$ and $\mathrm{C}$. 